OPEN ACCESS

Edited by: Eleonora Molinaro, University of Pisa, Italy

Reviewed by: Carla Gambale, University of Pisa, Italy Michael Cordes, University of Erlangen Nuremberg, Germany

${ }^{*}$ Correspondence: Chengcheng Niu niuchengcheng@csu.edu.cn

${ }^{\dagger}$ These authors have contributed equally to this work

Specialty section: This article was submitted to Cancer Endocrinology, a section of the journal Frontiers in Endocrinology

Received: 26 September 2021 Accepted: 15 November 2021 Published: 08 December 2021

Citation:

Fang F, Gong Y, Liao L, Ye F, Zuo Z, Qi Z, Li X and Niu C (2021)

Value of Contrast-Enhanced Ultrasound in Partially Cystic Papillary Thyroid Carcinomas.

Front. Endocrinol. 12:783670. doi: 10.3389/fendo.2021.783670

\section{Value of Contrast-Enhanced Ultrasound in Partially Cystic Papillary Thyroid Carcinomas}

\author{
Fengkai Fang ${ }^{1 \dagger}$, Yi Gong ${ }^{2 \dagger}$, Liyan Liao ${ }^{3}$, Fei Ye ${ }^{2}$, Zhongkun Zuo ${ }^{2}$, Zhang Qi ${ }^{1}$, \\ Xiaodu $\mathrm{Li}^{1}$ and Chengcheng Niu ${ }^{1 *}$ \\ ${ }^{1}$ Department of Ultrasound Diagnosis, The Second Xiangya Hospital, Central South University, Changsha, China, \\ ${ }^{2}$ Department of Thyroid Surgery, The Second Xiangya Hospital, Central South University, Changsha, China, ${ }^{3}$ Department of \\ Pathology, The Second Xiangya Hospital, Central South University, Changsha, China
}

Partially cystic papillary thyroid carcinomas (PCPTCs) are rarely reported papillary thyroid carcinomas (PTCs) and are usually misdiagnosed as benign nodules. The objective of this study was to provide the various sonographic characteristics of partially cystic thyroid nodules for differentiation between malignant and benign nodules, including those for conventional ultrasound (US) and contrast-enhanced ultrasound (CEUS). Twenty-three PCPTC patients and 37 nodular goiter patients were enrolled in this study. We evaluated the size, cystic percentage, solid echogenicity, calcification, vascularity, and CEUS parameters for each nodule. The final diagnosis of all patients was confirmed via surgery. Univariate analysis demonstrated that compared with benign nodular goiters, PCPTCs more frequently presented with calcification, hypoechogenicity of the solid part, hypoenhancement, heterogeneous enhancement, centrifugal perfusion, peak intensity index $<1$, time to peak index $\geq 1$, and area under the curve index $<1$ on preoperative US and CEUS. Binary logistic regression analysis demonstrated that heterogeneous enhancement, centrifugal perfusion, and peak intensity index $<1$ are independent CEUS characteristics related to malignant PCPTCs and can be used for their differentiation from benign nodular goiters (all $p<0.05$ ). Our study indicated that preoperative CEUS characteristics may serve as a useful tool to distinguish malignant PCPTCs from benign thyroid nodules.

Keywords: thyroid carcinomas, partially cystic thyroid nodules, thyroid ultrasonography, partially cystic papillary thyroid carcinomas (PCPTCs), contrast-enhanced ultrasound (CEUS)

\section{INTRODUCTION}

Thyroid carcinomas have a mostly solid composition, but those with predominant cystic changes ( $>50 \%$ of the nodule) can be observed in $2.5 \%-6.0 \%$ of all thyroid carcinoma cases $(1,2)$. In a prospective study, 213 partially cystic thyroid nodules in 196 patients who had consecutively undergone prospective sonographic diagnosis and ultrasonography-guided fine-needle aspiration biopsy (US-FNAB) were included, and the rate of malignancy for partially cystic thyroid nodules was $5.2 \%$ (3). Thyroid nodules can be classified as cystic or almost completely cystic, spongiform, mixed cystic and solid, solid, or almost completely solid according to their composition as 
ascertained by ultrasonography (4). However, to our knowledge, there are few studies that have investigated sonographic features as predictors for the diagnosis of malignant partially cystic thyroid nodules.

US-FNAB is the preferred method for the preoperative diagnosis of benign and malignant thyroid lesions. Current guidelines consider a size $\geq 1 \mathrm{~cm}$ (in nodules with high suspicion), $1.5-2.0 \mathrm{~cm}$ (in nodules with any suspicious US features), or $2.0 \mathrm{~cm}$ (in nodules without any suspicious US features) as a criterion for US-FNAB, regardless of the cystic portion (5). However, US-FNAB has a high rate of nondiagnostic and false-negative results for the diagnosis of partially cystic thyroid nodules (6).

Contrast-enhanced ultrasound (CEUS), as a relatively novel ultrasound (US) technique, has great significance value in the diagnosis of collapsing benign cystic or predominantly cystic thyroid nodules when combined with clinical history according to the 2020 Chinese guidelines for ultrasound malignancy risk stratification of thyroid nodules $(7,8)$. However, very few published studies have reported the use of CEUS for predominant cystic thyroid carcinomas. To our knowledge, this is the first article describing the CEUS features and corresponding histopathology of PCPTCs. Hence, the aim of the present study was to provide CEUS characteristics for PCPTCs in order to distinguish them from benign nodules.

\section{MATERIALS AND METHODS}

\section{Patients}

The study was approved by the Ethical Committee of the Second Xiangya Hospital of Central South University in China and was performed in accordance with the Declaration of Helsinki for human studies. The requirement of informed consent from human subjects is sometimes waived by institutional review boards (IRBs) for protocols that include a retrospective review of images acquired for clinical diagnostic purposes. From June 2017 to August 2021, 27 partially cystic thyroid carcinoma patients who received conventional US and CEUS examinations were retrospectively enrolled in this case-control study. The inclusion criteria were as follows: 1) patients with mixed echoic thyroid nodules that were confirmed as PTCs with partial cystic degeneration by pathologic examinations after surgery; 2) no invasive procedure such as thyroid surgery or FNA was previously performed. Four patients were excluded because they had different types of thyroid cancers: two medullary thyroid carcinomas and two follicular carcinomas. For patients with multifocal PTCs, only the largest was selected. Finally, 23 patients with 23 PCPTCs were included in this study. In addition, from August 2017 to August 2021, 37 patients with 37 mixed echoic thyroid nodules who received conventional US and CEUS examinations were recruited for this study as a control group. The inclusion criteria were as follows: 1) patients with mixed echoic thyroid nodules that were confirmed to be nodular goiters by pathologic examination after surgery; 2) no invasive procedure such as thyroid surgery or FNA was previously performed. For patients with multifocal thyroid nodules, only the largest was selected. Finally, 37 patients with 37 nodular goiters were included in this study. Ultimately, 60 mixed echoic thyroid nodules in 60 patients were enrolled in the study.

\section{Conventional US}

A Siemens Acuson S3000 US scanner (Siemens Medical Solutions, Mountain View, CA, USA) equipped with 9L4 (4-9 $\mathrm{MHz})$ and $18 \mathrm{~L} 6(6-18 \mathrm{MHz})$ linear array transducers was used for conventional US, and a 9L4 linear array transducer was used for CEUS. All examinations were performed by the same operator with 15 years of experience in thyroid ultrasound diagnosis and 10 years of experience in performing CEUS to prevent bias from different operators and to ensure optimized image quality. All selected thyroid nodules were evaluated by conventional B-mode US and color-Doppler US for the following US features: size (the largest diameter), cystic percentage $(\geq 50 \%$ or $<50 \%)$, solid part echogenicity (hypoechogenicity or isoechogenicity), calcification (present or absent), and internal vascularity (present or absent).

The nodules were classified according to the Thyroid Imaging Reporting and Data System (TI-RADS) proposed by Kwak et al. (9). According to that classification, five US suspicious features (solid component, hypoechogenicity or marked hypoechogenicity, microlobulated or irregular margins, taller-than-wide shape, and presence of microcalcifications) were applied to categorize the thyroid nodules: TI-RADS score 3 (no suspicious US features), $4 \mathrm{a}$ (one suspicious US feature), $4 \mathrm{~b}$ (two suspicious US features), 4c (three or four suspicious US features), and 5 (five suspicious US features). In this study, hypoechogenicity was applied for the solid part of these mixed echoic thyroid nodules.

\section{CEUS and Analysis}

CEUS was performed using contrast pulsed sequencing (CPS) technology [mechanical index $(\mathrm{MI})=0.07]$. SonoVue (Bracco, Italy) was injected intravenously as a bolus of $3.0 \mathrm{ml}$ via a 20 gauge antecubital vein cannula, followed by a saline flush of $5 \mathrm{ml}$, with the timer started simultaneously. Thyroid nodule imaging lasted at least $60 \mathrm{~s}$. The CEUS videos were digitally recorded and analyzed with CEUS software (Contrast Dynamics, Mountain View, CA, USA). Time-intensity curves (TICs) of the thyroid within selected regions of interest (ROIs) were acquired, and the contrast enhancement features of thyroid nodules were applied according to our previous study (10). After comparison with the surrounding thyroid parenchymal enhancement, the contrast enhancement features were classified as follows: enhancement type (hyperenhancement, isoenhancement, hypoenhancement), perfusion pattern (centripetal perfusion, the perfusion of microbubbles from the periphery to the center of nodule; centrifugal perfusion, the perfusion of microbubbles from the center to the periphery of nodule), enhancement uniformity (homogeneous, the microbubbles were evenly distributed; heterogeneous, the microbubbles were unevenly distributed), peak intensity (PI; expressed as a percentage), time to peak (TP; expressed in seconds), and area under the curve (AUC; expressed in percentage by seconds). The PI, TP, and AUC of the nodules are reported as indices by the ratio of the ROI of the 
nodules to the ROI of the thyroid parenchymal tissue. PI index represents the ratio of the quantitative values of peak intensity of the nodule to the quantitative values of thyroid parenchymal tissue. If the average quantitative values of nodule were higher or equal to that of the thyroid parenchymal tissue, the PI index was expressed as $\geq 1$; if this was not the case, the PI index was expressed as $<1$. Similarly, TP index $\geq 1$ meant that the time to peak of the nodule was slower or equal to that of the thyroid parenchymal tissue; AUC index $\geq 1$ meant that the area under the curve of the nodule was higher or equal to that of the thyroid parenchymal tissue.

\section{Reference Standard}

FNA Bethesda cytology (BC) diagnoses were divided into six categories according to the Bethesda System (5). The histopathological results after surgery were used as the reference standard for the final diagnosis of PTCs or nodular goiters.

\section{Statistical Analysis}

The statistical analysis was performed with SPSS version 21.0 software (SPSS, Chicago, IL, USA). Continuous data are presented as the mean and standard deviation (SD) and compared by the independent t-test. Categorical data were presented as percentages and analyzed by the chi-square test. Binary logistic regression was used to assess significant CEUS features and their independent association with malignant partially cystic thyroid nodules. The sensitivity, specificity, accuracy, positive predictive value (PPV), and negative predictive value (NPV) of TI-RADS for differentiation between benign and malignant thyroid nodules were calculated. A statistically significant difference was determined when $\mathrm{p}<0.05$.

\section{RESULTS}

A total of 60 patients with 60 partially cystic thyroid nodules (23 malignant and 37 benign) were included in the analysis. For malignant thyroid nodules, the FNA cytology diagnoses for 23 nodules were as follows: one (4.3\%) nodule was BC 3 (atypia or follicular lesion of undetermined significance), three (13.1\%) nodules were BC 4 (follicular neoplasm or suspicious for a follicular neoplasm), seven (30.4\%) nodules were BC 5 (suspicious for malignancy), and 12 (52.2\%) nodules were BC 6 (malignant). All nodules were confirmed as PTC by pathologic examinations after surgery. For benign thyroid nodules, 16 patients had malignant-looking thyroid nodules on the other thyroid lobe, and FNA cytology was carried out for the malignant thyroid nodules. Thus, 16 patients with PTCs on the other thyroid lobe were confirmed by pathologic examinations after total thyroidectomy; 16 benign thyroid nodules on this thyroid lobe were also confirmed by histopathology. The FNA cytology diagnoses for the other 21 nodules were as follows: six (28.6\%) nodules were BC 3, and 15 (71.4\%) were BC 2 (benign).

The clinical characteristics of the patients are outlined in Table 1. The average ages of PCPTC patients and nodular goiter patients were $41.74 \pm 9.99$ years (range: $29-63$ years) and $51.92 \pm$ 9.55 years (range: 25-68 years), respectively, and PCPTC patients were younger than nodular goiter patients in this study $(\mathrm{p}<0.05)$. Male patients constituted $26.1 \%$ of PCPTC patients and $5.4 \%$ of nodular goiter patients; thus, female patients accounted for a larger proportion of nodular goiters than PCPTCs $(\mathrm{p}<0.05)$. Thirteen $(56.5 \%)$ PCPTC patients and $31(83.8 \%)$ nodular goiter patients had multifocal thyroid nodules, and nodular goiter patients had many more thyroid nodules per patient than PCPTC patients in this study $(\mathrm{p}<0.05)$.

The US characteristics of partially cystic thyroid nodules are outlined in Table 2. The mean diameters were $29.35 \pm 12.21 \mathrm{~mm}$ (range: 9-51 mm) for malignant partially cystic thyroid nodules and $32.97 \pm 14.39 \mathrm{~mm}$ (range: $10-68 \mathrm{~mm}$ ) for benign partially cystic thyroid nodules. In the malignant thyroid nodule group, six (26.1\%) nodules had cystic percentage greater than 50\% (Figure 1), 21 (91.3\%) nodules had calcifications (Figure 1), 18 (78.3\%) nodules exhibited hypoechogenicity in the solid part of the partially cystic nodules (Figure 1), and five (21.7\%) nodules exhibited isoechogenicity in the solid part. Seventeen (73.9\%) nodules had internal blood flow. For the CEUS parameters, 10 (43.5\%) nodules exhibited hypoenhancement (Figure 2), and 22 (95.7\%) nodules had heterogeneous enhancement (Figure 2), which meant that the microbubbles in the majority of the nodules were unevenly distributed. Twenty (87.0\%) nodules had a centrifugal perfusion pattern, and three nodules (13.0\%) had a centripetal perfusion pattern (Figure 2), indicating that most of the nodules received a perfusion of microbubbles from the center to the periphery. The quantitative CEUS parameters showed that $14(60.1 \%)$ nodules had a PI index $<1$ (Figure 2), 14 (60.9\%) nodules had a TP index $\geq 1$, and 21 (91.3\%) nodules had an AUC index $<1$ (Figure 2). In the benign thyroid nodule group, $15(40.5 \%)$ nodules had a cystic percentage greater than $50 \%, 29(78.4 \%)$ nodules had an absence of calcification, 15 (40.5\%) nodules exhibited hypoechogenicity in the solid part, and 22 (59.5\%) nodules exhibited isoechogenicity in the solid part. Twenty-three $(62.2 \%)$ nodules had internal blood flow. For the CEUS parameters, 31 (83.8\%) nodules exhibited hyperenhancement or isoenhancement, 30 (81.1\%) nodules had uniform enhancement, and 34 (91.9\%) nodules had a centripetal

TABLE 1 | Clinical characteristics of the PCPTCs and nodular goiters.

\begin{tabular}{lccc}
\hline Characteristics & PCPTCs $\mathbf{( n = 2 3 )}$ & Nodular goiter $(\mathbf{n}=\mathbf{3 7})$ & $\mathbf{p}$ Value \\
\hline Age (years) & $41.74 \pm 9.99$ & $51.92 \pm 9.55$ & $0.000^{\star}$ \\
Male sex & $6(26.1)$ & $2(5.4)$ & $0.045^{\star}$ \\
Multifocality & $13(56.5)$ & $31(83.8)$ & $0.020^{\star}$ \\
\hline
\end{tabular}

* $p<0.05$ was considered a significant difference.

PTPTC, partially cystic papillary thyroid carcinoma. 
TABLE 2 | Ultrasound characteristics of the PCPTCs and nodular goiters.

\begin{tabular}{|c|c|c|c|}
\hline Characteristics & PCPTCs $(n=23)$ & Nodular goiter $(n=37)$ & p Value \\
\hline \multicolumn{4}{|c|}{ Conventional US parameters } \\
\hline Size (mm) & $29.35 \pm 12.21$ & $32.97 \pm 14.39$ & 0.320 \\
\hline$\geq 50 \%$ & $6(26.1)$ & $15(40.5)$ & \\
\hline$<50 \%$ & 17 (73.9) & $22(59.5)$ & \\
\hline Calcification & & & $0.000^{*}$ \\
\hline Present & $21(91.3)$ & $8(21.6)$ & \\
\hline Hypoechogenicity & $18(78.3)$ & $15(40.5)$ & \\
\hline Isoechogenicity & $5(21.7)$ & $22(59.5)$ & \\
\hline Internal vascularity & & & 0.408 \\
\hline Present & $17(73.9)$ & $23(62.2)$ & \\
\hline Absent & $6(26.1)$ & $14(37.8)$ & \\
\hline \multicolumn{4}{|l|}{ CEUS parameters } \\
\hline Homogeneous & $1(4.3)$ & $30(81.1)$ & \\
\hline Heterogeneous & $22(95.7)$ & 7 (18.9) & \\
\hline Perfusion & & & $0.000^{*}$ \\
\hline Centripetal & $3(13.0)$ & $34(91.9)$ & \\
\hline Centrifugal & $20(87.0)$ & $3(8.1)$ & \\
\hline $\mathrm{Pl}$ index & & & $0.000^{*}$ \\
\hline$\geq 1$ & $9(39.1)$ & $31(83.8)$ & \\
\hline$<1$ & $14(60.9)$ & $6(16.2)$ & \\
\hline TP index & & & $0.001^{*}$ \\
\hline$\geq 1$ & $14(60.9)$ & $7(18.9)$ & \\
\hline$<1$ & $9(39.1)$ & $30(81.1)$ & \\
\hline AUC index & & & $0.000^{*}$ \\
\hline$\geq 1$ & $2(8.7)$ & $28(75.7)$ & \\
\hline
\end{tabular}

${ }^{*} p<0.05$ was considered a significant difference.

PI, peak intensity; TP, time to peak; TP, time to peak time; AUC, area under the curve; PCPTC, partially cystic papillary thyroid carcinoma.

perfusion pattern. The quantitative CEUS parameters showed that 31 (83.8\%) nodules had a PI index $\geq 1,30$ (81.1\%) nodules had a TP index $<1$, and $28(75.7 \%)$ nodules had an AUC index $\geq 1$. The univariate analysis indicated that the PCPTCs more frequently presented with calcification, hypoechogenicity of the solid part, hypoenhancement, heterogeneous enhancement, centrifugal perfusion, PI index $<1$, TP index $\geq 1$, and AUC index $<1$ for preoperative US and CEUS compared to benign thyroid nodules (all $\mathrm{p}<0.05$ ).

For evaluation of the value of CEUS parameters in PCPTCs, a binary logistic regression analysis was performed for all of the statistically significant CEUS variables $(\mathrm{p}<0.05)$. The results indicated that enhancement uniformity $(\mathrm{B}=4.080$, $\mathrm{OR}=59.166$, $95 \% \mathrm{CI}=1.928-1,815.846, \mathrm{p}=0.020)$, centrifugal perfusion $(\mathrm{B}=$ $4.502, \mathrm{OR}=90.157,95 \% \mathrm{CI}=4.443-1,829.637, \mathrm{p}=0.003)$, and PI index $<1(B=5.515, O R=248.279,95 \% \mathrm{CI}=1.655-37241.707$, $\mathrm{p}=0.031)$ were independent characteristics related to the PCPTC nodules that could be used to differentiate them from benign nodular goiters (Table 3 ).

Heterogeneous enhancement, centrifugal perfusion, and PI index $<1$ were independent characteristics related to PCPTCs that could be used to differentiate them from benign thyroid nodes; therefore, we chose these CEUS parameters for the diagnosis of PCPTCs. If the thyroid nodule had more than 2 $(\geq 2)$ of the above three CEUS characteristics, the thyroid nodule was classified as a malignant thyroid nodule; if the thyroid nodule had less than $2(<2)$ of the above three CEUS characteristics, the thyroid nodule was classified as a benign thyroid nodule. Then, the combination diagnosis of Kwak TI-RADS classification and CEUS characteristics was calculated. If the thyroid nodule had a Kwak TI-RADS $4 \mathrm{~b}$ score and/or CEUS $\geq 2$, the thyroid nodule was classified as a malignant thyroid nodule; if the thyroid nodule had a Kwak TI-RADS 4a score and/or CEUS $<2$, the thyroid nodule was classified as a benign thyroid nodule. The diagnostic performance of the different methods for differentiation between benign and malignant thyroid nodules is outlined in Table 4. The Kwak TI-RADS with a cutoff value of $4 \mathrm{a} / 4 \mathrm{~b}$ score achieved an $\mathrm{Az}$ value of 0.851 , with an accuracy of $86.7 \%$ (52/60). The CEUS characteristics with a cutoff value of CEUS $\geq 2$ achieved an $\mathrm{Az}$ value of 0.924 , with an accuracy of $91.7 \%(55 / 60)$. The combination of Kwak TI-RADS and CEUS achieved an Az value of 0.897 , with a cutoff value of $4 \mathrm{a} / 4 \mathrm{~b}$ or CEUS $\geq 2$, and had an accuracy of $88.3 \%$ (53/60), which was better than that of Kwak TI-RADS. However, there was no significant difference with respect to diagnostic accuracy for differentiation between benign and malignant thyroid nodules among all groups $(\mathrm{p}>0.05)$. 

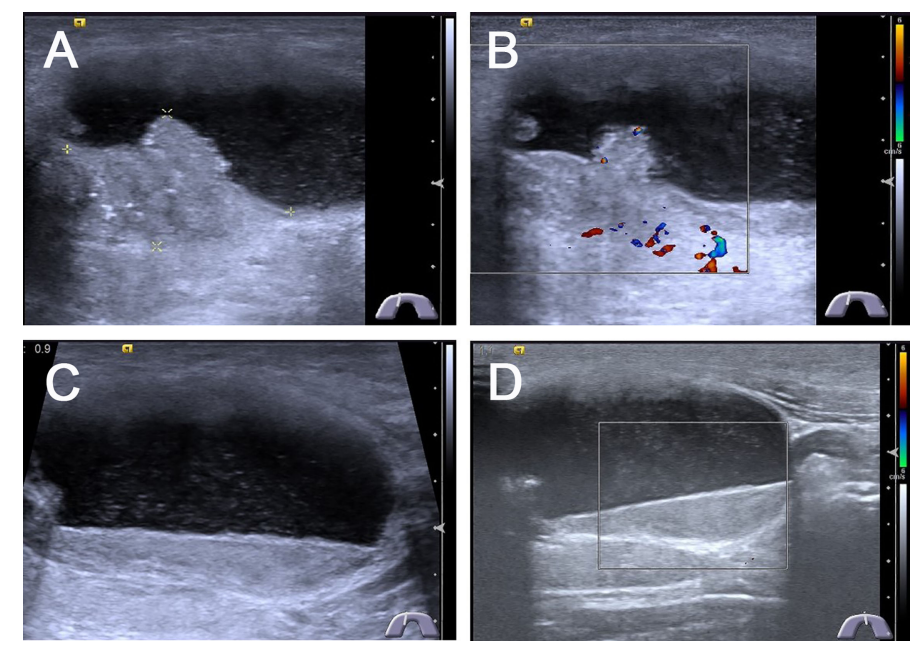

FIGURE 1 | Conventional ultrasonography images of predominantly cystic papillary thyroid carcinoma. (A) Longitudinal gray-scale sonography revealed a predominantly cystic $4.2 \times 2.4 \times 2.7 \mathrm{~cm}^{3}$ thyroid nodule with a little solid portion abutted on the side of the cyst wall in the thyroid isthmus, the cystic portion was more than $90 \%$ of the thyroid node. (B) CDFI showed poor blood flow signals in the solid portion of the thyroid nodule. (C) Most portions of this thyroid node were cystic with a lot of mobile silt-like isoechoic substance. (D) CDFI showed no blood flow signals in the silt-like isoechoic substance.

Of 23 PCPTCs patients, $14(60.1 \%)$ patients had central cervical lymph node metastasis. Histopathological examination of samples using hematoxylin and eosin (H\&E) staining showed a predominantly PCPTC with a cystic percentage greater than 90\% (Figure 3), revealing the thick cystic wall of the mass (3-6 $\mathrm{mm}$ ) (Figures 3A, B) and the solid part abutting on the base side of the cyst wall (Figures 3C, D). Many nipple-like bulges were seen on both the cyst wall and the solid part. Meanwhile, some nipple-like bulges (black arrows indicated) were also observed in the central lymph nodes (Figures 3E, F), which indicated that those lymph nodes were metastatic for thyroid carcinoma.

\section{DISCUSSION}

Partially cystic thyroid nodules are common on ultrasonography and are considered to be a result of the cystic degeneration of either neoplastic or non-neoplastic nodules (11). Although partially cystic thyroid nodules are traditionally interpreted as having a low risk of malignancy, some studies have reported that the frequency of malignancy among partially cystic thyroid nodules varies from $5.2 \%$ to $17.6 \%(3,6,12,13)$. A study by Kim et al. (14) compared the disease-free survival of 553 PTCs with cystic changes according to the percentage of cystic component (two groups: $25 \%$ or 50\%) of the thyroid nodules and found that the proportion of the cystic component in PTCs did not affect disease-free survival. In this study, 56 patients (10.1\%) were confirmed to have tumor recurrence within the follow-up period, while the independent predictors of recurrence were pathologic size, male sex, and lymph node metastasis. Therefore, it is vital to differentiate malignant thyroid nodules from benign partially cystic thyroid nodules using preoperative ultrasonography and/or US-FNAB.
Some studies have reported the conventional sonographic features of partially cystic thyroid nodules associated with malignancy. Lee et al. (12) reported that partially cystic thyroid nodules with a predominantly solid component (solid portion was $>50 \%$ ), an eccentrically placed solid component, and the presence of microcalcifications were all associated with malignancy. Kim et al. (3) reported that partially cystic thyroid nodules had an eccentric solid portion with an acute angle, and microcalcifications were significantly associated with malignancy. Park et al. (15) reported the malignant sonographic features of an entire partially cystic thyroid nodule and its internal solid portion. A taller-thanwide shape and spiculated or microlobulated margins were associated with malignancy in partially cystic thyroid nodules. Furthermore, an eccentric solid configuration, non-smooth margin, hypoechogenicity, and microcalcifications of the internal solid portion were significantly associated with malignancy. In our study, most PCPTCs had an eccentric solid portion with microcalcifications, which was consistent with recent reports (3, $12,15)$. However, the acute angle of the internal solid portion, the taller-than-wide shape, and the spiculated or microlobulated margin of the entire nodule were not shown in our study (15). In a study by Henrichsen et al. (1), 360 malignant thyroid nodules that had been surgically removed were analyzed, nine $(2.5 \%)$ of which were $51 \%-100 \%$ cystic. Of the nine malignancies (cystic portion $>50 \%$ ), four (44\%) demonstrated increased vascularity either in a nodule or in thickened walls (1). In our study, some PCPTCs showed poor blood flow signals in the internal solid portion of the thyroid nodule, which was similar to the Color Doppler Flow Imaging (CDFI) findings of solid PTCs reported by other studies $(10,16)$. However, there are very few reports regarding the CEUS findings of malignant partially cystic thyroid nodules.

CEUS, as a novel technique for detecting microvessels of tissues, has been widely applied in improving the diagnostic 

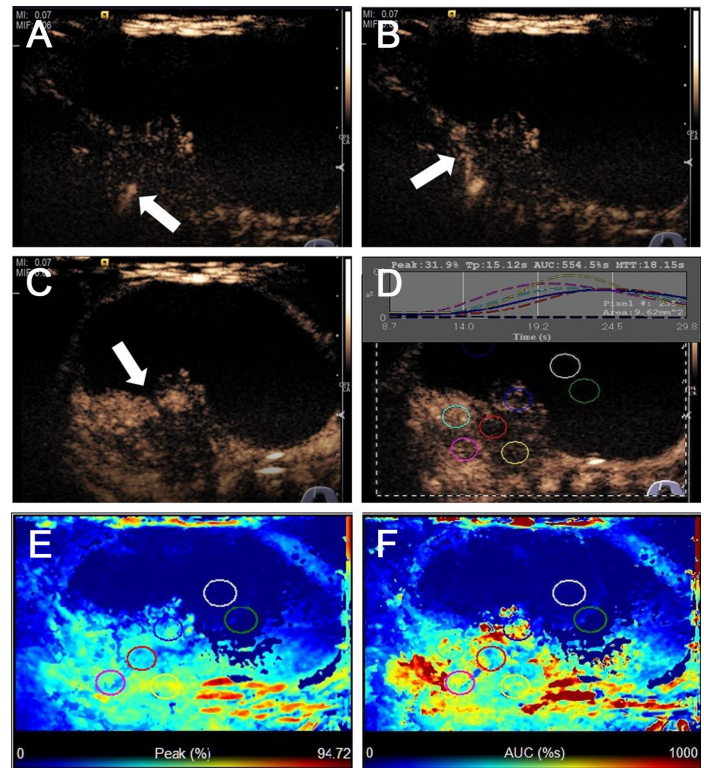

FIGURE 2 | CEUS images of predominantly cystic papillary thyroid carcinoma. (A) CEUS image showed a slight enhancement from the bottom of the solid portion at $9 \mathrm{~s}$. (B) The enhancement from the bottom to the periphery of the solid portion at $15 \mathrm{~s}$. (C) All the solid portions of this nodule heterogeneously enhanced and reached its peak [time to peak (TTP)] at $23 \mathrm{~s}$. (D) TICs displayed the wash-in time of $9 \mathrm{~s}$, TTP of $15 \mathrm{~s}$, PI of $31.9 \%$, and AUC of $554.5 \% \mathrm{~s}$ for the solid portion of this thyroid nodule, and the cystic portion of the nodule has no enhancement. (E) The parametric color map showed that the solid portion was almost a majority of green with a little blue, the cystic portion was totally blue, which indicated that the Pls for the center of the solid portion was almost equal to those of the periphery of the solid portion. (F) The parametric color map showed the solid portion was heterogeneous with a mixture of green, yellow, and red, and the cystic portion was totally blue, which indicated that the AUC for the center of the solid portion was lower than those of the periphery of the solid portion.

accuracy of thyroid nodules. Deng et al. (17) reported that hypoenhancement on CEUS correlated highly with a malignant diagnosis of thyroid nodules (sensitivity: $82.1 \%$, specificity: $84.9 \%$, accuracy: $84.0 \%$, PPV: $71.9 \%$, and NPV: $91.0 \%$ ). Ma et al. (18) found that heterogeneous enhancement on CEUS showed the best diagnostic performance for papillary thyroid microcarcinoma, with the highest PPV of $88.0 \%$, an accuracy of $83.7 \%$, and a relatively high specificity of $83.9 \%$. However, mixed cystic nodules or almost cystic nodules (cystic portion $>75 \%$ ) were excluded from previous studies due to the demand for elastography $(10,17,18)$. In our study, $43.5 \%$ of PCPTCs showed hypoenhancement, $95.7 \%$ of PCPTCs showed heterogeneous enhancement, and $87 \%$ of PCPTCs exhibited centrifugal perfusion, which is inconsistent with the CEUS enhancement of solid PTCs reported in previous studies $(10,19,20)$. Compared with benign nodular goiters, PCPTCs more frequently had hypoenhancement, heterogeneous enhancement, centrifugal perfusion, PI index $<1$, TP index $\geq 1$, and AUC index $<1$ on preoperative CEUS. Binary logistic regression analysis demonstrated that heterogeneous enhancement, centrifugal perfusion, and PI index $<1$ are independent CEUS characteristics related to malignant PCPTCs that can be used to differentiate them from benign nodular goiters (all $\mathrm{p}<0.05$ ). This may be due to increased neovascularization in the malignant nodules; however, the invasive growth characteristics of malignant nodules also destroyed the new blood vessels, formed small tumor thrombi in the necrotic vessels, and led to the occlusion of small blood vessels. Therefore, the degree of CEUS enhancement not only is related to the number of blood vessels but also depends on the state of internal blood vessel function. Especially in PCPTCs with cystic percentage greater than $90 \%$, the solid portion on the cyst wall of the thyroid nodule had few blood vessels around the papillary protrusion in the cyst wall based on the H\&E histopathological staining results, which was consistent with the heterogeneous hypoenhancement on CEUS. These results may be explained by the fact that the inner solid portion of the malignant nodule was often located at the base of the papillary protrusion in the cyst wall, and the blood vessels around the lesion easily invaded outward, resulting in ischemic necrosis of the inner solid portion near the side of the cystic portion and continuous cystic degeneration. Furthermore, the inner solid portion of the malignant nodule was located at the base of the papillary protrusion in the cyst wall, and the blood vessels around the lesion easily invaded outward, which may lead to central cervical lymph node metastasis.

This study had several limitations. First, an unavoidable selection bias may have existed, and some patients with suspicious malignancies might not have been enrolled in this study because they did not have surgery. Second, the nodule sizes studied for comparison between the two groups ranged from a few millimeters to tens of millimeters; these size differences might affect the US characteristics of PCPTCs. Third, all conventional US and CEUS examinations were performed by a single experienced operator, which means that the study was not 
TABLE 3 | Multivariate logistic regression analysis of CEUS characteristics related to PCPTCs distinguishing from nodular goiters.

\begin{tabular}{|c|c|c|c|c|}
\hline Characteristics & Partial regression coefficient, $\beta$ & Odds ratio & $95 \%$ Confidence interval & p Value \\
\hline Heterogeneous enhancement & 4.080 & 59.166 & $1.928-1,815.846$ & $0.020^{*}$ \\
\hline Centrifugal perfusion & 4.502 & 90.157 & $4.443-1,829.637$ & $0.003^{*}$ \\
\hline $\mathrm{Pl}$ index $<1$ & 5.515 & 248.279 & $1.655-37,241.707$ & $0.031^{*}$ \\
\hline
\end{tabular}

${ }^{*} p<0.05$ was considered a significant difference.

PCPTC, partially cystic papillary thyroid carcinoma.

TABLE 4 | Diagnostic performance for discrimination between PCPTCs and nodular goiters.

\begin{tabular}{|c|c|c|c|c|c|c|c|}
\hline Methods & Cutoff score & Sensitivity, \% & Specificity, \% & Accuracy, \% & PPV, \% & NPV, \% & $\mathrm{Az}(95 \% \mathrm{Cl})$ \\
\hline TI-RADS & $4 a / 4 b$ & 78.3 & 91.9 & 86.7 & 85.7 & 87.2 & $0.851(0.738-0.963)$ \\
\hline CEUS & CEUS $\geq 2$ & 95.7 & 89.2 & 91.7 & 84.6 & 97.1 & $0.924(0.848-1.000)$ \\
\hline TI-RADS+CEUS & $4 a / 4 b$ or CEUS $\geq 2$ & 95.7 & 83.8 & 88.3 & 78.6 & 96.9 & $0.897(0.811-0.984)$ \\
\hline
\end{tabular}

TIRADS, Thyroid Imaging Reporting and Data System; CEUS, contrast-enhanced ultrasound; PPV, positive predictive value; NPV, negative predictive value; CI, confidence interval; PCPTC, partially cystic papillary thyroid carcinoma.
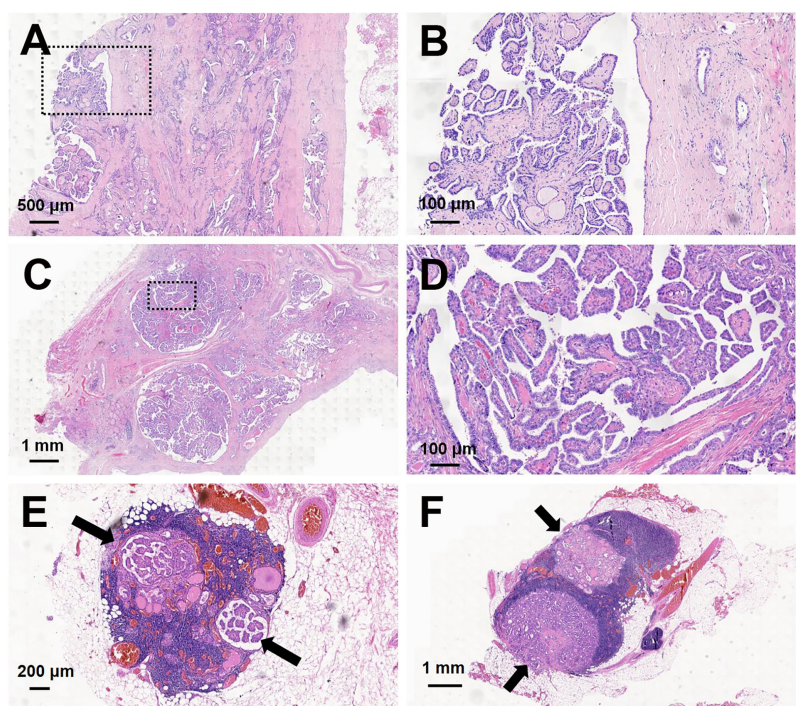

FIGURE 3 | Hematoxylin and eosin (H\&E) staining of predominantly cystic papillary thyroid carcinoma and central cervical lymph nodes. The thick cystic wall of the mass showed a lot of nipple-like bulges: (A) magnification, $\times 20$; (B) magnification, $\times 100$. The solid portion of the mass showed a lot of nipple-like bulges: (C) magnification, $\times 10$; (D) magnification, $\times 100$. Some nipple-like bulges (black arrows indicated) were shown in two central cervical lymph nodes: (E) magnification, $\times 25$; (F) magnification, $\times 10$.

operator independent. Because subjective interference caused by other examiners might exist, further studies with more operators performing each examination are needed. Fourth, this was a small-scale retrospective study, which may have caused the inaccurate evaluation of the effect on CEUS parameters by the different nodule sizes of the two groups. Thus, a large-scale prospective study is needed to clarify these findings in the future.

\section{CONCLUSION}

PCPTCs are extremely rare cystic PTCs, and very few studies describe their ultrasonographic imaging features and related pathologic findings. On preoperative US and CEUS, PCPTCs more frequently presented with calcification, hypoechogenicity of the solid part, hypoenhancement, heterogeneous enhancement, centrifugal perfusion, PI index $<1$, TP index $\geq 1$, and AUC index $<1$ compared with benign nodular goiters. Binary logistic regression analysis demonstrated that heterogeneous enhancement, centrifugal perfusion, and PI index $<1$ are independent CEUS characteristics related to malignant PCPTCs that can be used to differentiate them from benign nodular goiters. Thus, preoperative CEUS characteristics may serve as a useful tool to distinguish malignant PCPTCs from benign thyroid nodules and thus effectively supplement conventional US. 


\section{DATA AVAILABILITY STATEMENT}

The raw data supporting the conclusions of this article will be made available by the authors without undue reservation.

\section{ETHICS STATEMENT}

The studies involving human participants were reviewed and approved by the Ethical Committee of the Second Xiangya Hospital, Central South University. The patients/participants provided their written informed consent to participate in this study. Written informed consent was obtained from the individual(s) for the publication of any potentially identifiable images or data included in this article.

\section{REFERENCES}

1. Henrichsen TL, Reading CC, Charboneau JW, Donovan DJ, Sebo TJ, Hay ID. Cystic Change in Thyroid Carcinoma: Prevalence and Estimated Volume in 360 Carcinomas. J Clin Ultrasound (2010) 38(7):361-6. doi: 10.1002/ jcu. 20714

2. Chan BK, Desser TS, McDougall IR, Weigel RJ, Jeffrey RB Jr. Common and Uncommon Sonographic Features of Papillary Thyroid Carcinoma. J Ultrasound Med (2003) 22(10):1083-90. doi: 10.7863/jum. 2003.22.10.1083

3. Kim DW, Lee EJ, In HS, Kim SJ. Sonographic Differentiation of Partially Cystic Thyroid Nodules: A Prospective Study. AJNR Am J Neuroradiol (2010) 31(10):1961-6. doi: 10.3174/ajnr.A2204

4. Tessler FN, Middleton WD, Grant EG, Hoang JK, Berland LL, Teefey SA, et al. ACR Thyroid Imaging, Reporting and Data System (TI-RADS): White Paper of the ACR TI-RADS Committee. J Am Coll Radiol (2017) 14(5):58795. doi: 10.1016/j.jacr.2017.01.046

5. Haugen BR, Alexander EK, Bible KC, Doherty GM, Mandel SJ, Nikiforov YE, et al. 2015 American Thyroid Association Management Guidelines for Adult Patients With Thyroid Nodules and Differentiated Thyroid Cancer: The American Thyroid Association Guidelines Task Force on Thyroid Nodules and Differentiated Thyroid Cancer. Thyroid (2016) 26(1):1-133. doi: 10.1089/ thy.2015.0020

6. Bellantone R, Lombardi CP, Raffaelli M, Traini E, De Crea C, Rossi ED, et al. Management of Cystic or Predominantly Cystic Thyroid Nodules: The Role of Ultrasound-Guided Fine-Needle Aspiration Biopsy. Thyroid (2004) 14 (1):43-7. doi: 10.1089/105072504322783830

7. Zhou J, Yin L, Wei X, Zhang S, Song Y, Luo B, et al. A. Vascular Ultrasound Group of the Society of Ultrasound in Medicine of the Chinese Medical, T. Chinese Artificial Intelligence Alliance for and U. Breast: 2020 Chinese Guidelines for Ultrasound Malignancy Risk Stratification of Thyroid Nodules: The C-TIRADS. Endocrine (2020) 70(2):256-79. doi: 10.1007/ s12020-020-02441-y

8. Peng Q, Niu C, Zhang Q, Zhang M, Chen S, Peng Q. Mummified Thyroid Nodules: Conventional and Contrast-Enhanced Ultrasound Features. J Ultrasound Med (2019) 38(2):441-52. doi: 10.1002/jum.14712

9. Kwak JY, Han KH, Yoon JH, Moon HJ, Son EJ, Park SH, et al. Thyroid Imaging Reporting and Data System for US Features of Nodules: A Step in Establishing Better Stratification of Cancer Risk. Radiology (2011) 260 (3):892-9. doi: 10.1148/radiol.11110206

10. Peng Q, Niu C, Zhang M, Peng Q, Chen S. Sonographic Characteristics of Papillary Thyroid Carcinoma With Coexistent Hashimoto's Thyroiditis: Conventional Ultrasound, Acoustic Radiation Force Impulse Imaging and Contrast-Enhanced Ultrasound. Ultrasound Med Biol (2019) 45(2):471-80. doi: 10.1016/j.ultrasmedbio.2018.10.020

11. Li W, Zhu Q, Jiang Y, Zhang Q, Meng Z, Sun J, et al. Partially Cystic Thyroid Nodules in Ultrasound-Guided Fine Needle Aspiration: Prevalence of

\section{AUTHOR CONTRIBUTIONS}

$\mathrm{CN}$ contributed to the conception and design of the work. FF and YG participated in the data analysis and article writing. LL, FY, $\mathrm{ZZ}, \mathrm{ZQ}$, and XL participated in the data collection and patients' follow-up. All authors contributed to the article and approved the submitted version.

\section{FUNDING}

This project was funded by the National Natural Science Foundation of China (81974267) and the Science and Technology Innovation Program of Hunan Province (2021RC3033).

Thyroid Carcinoma and Ultrasound Features. Med (Baltimore) (2017) 96 (46):e8689. doi: 10.1097/MD.0000000000008689

12. Lee MJ, Kim EK, Kwak JY, Kim MJ. Partially Cystic Thyroid Nodules on Ultrasound: Probability of Malignancy and Sonographic Differentiation. Thyroid (2009) 19(4):341-6. doi: 10.1089/thy.2008.0250

13. Garcia-Pascual L, Barahona MJ, Balsells M, del Pozo C, Anglada-Barcelo J, Casalots-Casado J, et al. Complex Thyroid Nodules With Nondiagnostic Fine Needle Aspiration Cytology: Histopathologic Outcomes and Comparison of the Cytologic Variants (Cystic vs. Acellular). Endocrine (2011) 39(1):33-40. doi: 10.1007/s12020-010-9409-2

14. Kim JY, Kim EK, Lee HS, Kwak JY. Conventional Papillary Thyroid Carcinoma: Effects of Cystic Changes Visible on Ultrasonography on Disease Prognosis. Ultrasonography (2014) 33(4):291-7. doi: 10.14366/ usg. 14028

15. Park JM, Choi Y, Kwag HJ. Partially Cystic Thyroid Nodules: Ultrasound Findings of Malignancy. Korean J Radiol (2012) 13(5):530-5. doi: 10.3348/ kjr.2012.13.5.530

16. Sun CY, Lei KR, Liu BJ, Bo XW, Li XL, He YP, et al. Virtual Touch Tissue Imaging and Quantification (VTIQ) in the Evaluation of Thyroid Nodules: The Associated Factors Leading to Misdiagnosis. Sci Rep (2017) 7:41958. doi: 10.1038/srep41958

17. Deng J, Zhou P, Tian SM, Zhang L, Li JL, Qian Y. Comparison of Diagnostic Efficacy of Contrast-Enhanced Ultrasound, Acoustic Radiation Force Impulse Imaging, and Their Combined Use in Differentiating Focal Solid Thyroid Nodules. PloS One (2014) 9(3):e90674. doi: 10.1371/journal.pone. 0090674PONE-D-13-30329[pii

18. Ma HJ, Yang JC, Leng ZP, Chang Y, Kang H, Teng LH. Preoperative Prediction of Papillary Thyroid Microcarcinoma Via Multiparameter Ultrasound. Acta Radiol (2017) 58(11):1303-11. doi: 10.1177/0284185117692167

19. Chen S, Niu C, Peng Q, Tang K. Sonographic Characteristics of Papillary Thyroid Carcinoma With Coexistent Hashimoto's Thyroiditis in the Preoperative Prediction of Central Lymph Node Metastasis. Front Endocrinol (Lausanne) (2021) 12:556851. doi: 10.3389/fendo. 2021.556851

20. Hong YR, Yan CX, Mo GQ, Luo ZY, Zhang Y, Wang Y, et al. Elastography, and Contrast Enhanced US Features of Papillary Thyroid Microcarcinoma Predict Central Compartment Lymph Node Metastases. Sci Rep (2015) 5:7748. doi: 10.1038/srep07748

Conflict of Interest: The authors declare that the research was conducted in the absence of any commercial or financial relationships that could be construed as a potential conflict of interest.

Publisher's Note: All claims expressed in this article are solely those of the authors and do not necessarily represent those of their affiliated organizations, or those of the publisher, the editors and the reviewers. Any product that may be evaluated in 
this article, or claim that may be made by its manufacturer, is not guaranteed or endorsed by the publisher.

Copyright (๑) 2021 Fang, Gong, Liao, Ye, Zuo, Qi, Li and Niu. This is an open-access article distributed under the terms of the Creative Commons Attribution License
(CC BY). The use, distribution or reproduction in other forums is permitted, provided the original author(s) and the copyright owner(s) are credited and that the original publication in this journal is cited, in accordance with accepted academic practice. No use, distribution or reproduction is permitted which does not comply with these terms. 